\title{
Epidemiological evidence on extra-medical use of prescription pain relievers: Transitions from newly incident use to dependence among 12-21 year olds in the United States using meta-analysis, 2002-13
}

Maria A. Parker, James C. Anthony

Background. When 12-to-21-year-olds start using prescription pain relievers extramedically, some of them transition into opioid dependence within 12 months after such use. Our main aim for this epidemiological research on 12-to-21-year-olds in the United States (US) is to estimate the risk of becoming a newly incident case of opioid dependence within 12 months after onset of using prescription pain relievers extra-medically (EMPPR). Methods. Meta-analyses from multiple independent replication samples now are possible, based upon nationally representative survey samples of US adolescents age 12-21 years. All 12-to-21-year-olds were sampled and recruited for the US National Surveys on Drug Use and Health, with standardized assessments of EMPPR use and opioid dependence (NSDUH, 2002-2013).

Results. Peak risk for a transition from start of EMPPR use to opioid dependence within 12 months is seen at mid-adolescence among 14 -to-15-year-olds (6.3\%, 8.7\% per year), somewhat earlier than peak risk for starting EMPPR use (seen for 16-to-19-year-olds at $4.1 \%, 5.9 \%$ per year). Applied to 12-to-21-year-olds in the US between 2002-2013, an estimated 8 million started using PPR extra-medically. Each year, roughly 42,000 to 58,000 transitioned into opioid dependence within 12 months after onset of such use.

Discussion. These epidemiological estimates for the US in recent years teach us to expect one transition into adolescent-onset opioid dependence within 12 months for every 11-16 newly incident EMPPR users, yielding perhaps 120 newly incident opioid dependent cases in need of practitioner attention or treatment services, each day of each year. This evidence can be used to motivate more effective public health prevention, outreach, and early intervention programs as might prevent or delay occurrence of EMPPR use and opioid dependence. 
2 Epidemiological evidence on extra-medical use of prescription pain relievers:

3 Transitions from newly incident use to dependence among 12-21 year olds in

4 the United States using meta-analysis, 2002-2013

5

6 Maria A. Parker ${ }^{1} \&$ James C. Anthony ${ }^{1}$

7

$8 \quad{ }^{1}$ Department of Epidemiology \& Biostatistics, College of Human Medicine, Michigan State

9 University, East Lansing, MI, United States

10

11 Corresponding Author:

12 James C. Anthony, $\mathrm{PhD}^{1}$

13 West Fee Hall, 909 Fee Road, East Lansing, MI 48824, United States

14 Email address: janthony@msu.edu 


\section{ABSTRACT}

16 Background. When 12-to-21-year-olds start using prescription pain relievers extra-medically,

17 some of them transition into opioid dependence within 12 months after such use. Our main aim

18 for this epidemiological research on 12-to-21-year-olds in the United States (US) is to estimate

19 the risk of becoming a newly incident case of opioid dependence within 12 months after onset of

20 using prescription pain relievers extra-medically (EMPPR).

21 Methods. Meta-analyses from multiple independent replication samples now are possible, based

22 upon nationally representative survey samples of US adolescents age 12-21 years. All 12-to-21-

23 year-olds were sampled and recruited for the US National Surveys on Drug Use and Health, with

24 standardized assessments of EMPPR use and opioid dependence (NSDUH, 2002-2013).

25 Results. Peak risk for a transition from start of EMPPR use to opioid dependence within 12

26 months is seen at mid-adolescence among 14-to-15-year-olds $(6.3 \%, 8.7 \%$ per year), somewhat

27 earlier than peak risk for starting EMPPR use (seen for 16-to-19-year-olds at 4.1\%, 5.9\% per

28 year). Applied to 12-to-21-year-olds in the US between 2002-2013, an estimated 8 million

29 started using PPR extra-medically. Each year, roughly 42,000 to 58,000 transitioned into opioid

30 dependence within 12 months after onset of such use.

31 Discussion. These epidemiological estimates for the US in recent years teach us to expect one

32 transition into adolescent-onset opioid dependence within 12 months for every 11-16 newly

33 incident EMPPR users, yielding perhaps 120 newly incident opioid dependent cases in need of

34 practitioner attention or treatment services, each day of each year. This evidence can be used to

35 motivate more effective public health prevention, outreach, and early intervention programs as

36 might prevent or delay occurrence of EMPPR use and opioid dependence. 


\section{BACKGROUND}

The United States continues to experience dramatic growth in numbers of overdoses and overdose deaths attributed to prescription pain relievers (PPR), a drug subtype that consists mainly of prescription-type opioid drug compounds with trade names such as Vicodin ${ }^{\text {, }}$ Lortab $\AA$, Lorcet ${ }^{\circledR}$, Darvocet ${ }^{\circledR}$, Darvon $\AA$, Tylenol ${ }^{\circledR}$ with Codeine, Percocet $\AA$, Percodan $\AA$, Tylox ${ }^{\circledR}$, and OxyContin ${ }^{\circledR}$ (United States Centers for Disease Control and Prevention, 2014). Supplement S1 provides a more comprehensive list of the compounds termed 'prescription pain relievers' in research of this type.

All too often, these overdoses are occurring after 12-to-21-year-olds have started to use PPR extra-medically [i.e., to get high and for related feelings and experiences, or otherwise outside the boundaries of a prescriber's intent, as defined in Anthony et al., 1994]. Hereinafter, PPR is the acronym for 'prescription pain reliever' and 'extra-medical prescription pain reliever' is designated by the abbreviation EMPPR. Sidebar 1 and Supplement S2 provide operational specifications for the more general concept of extra-medical drug use.

For the most part, growth in numbers of PPR overdoses in the United States (US) can be traced back to extra-medical use of these compounds as opposed to taking medicines exactly as prescribed (Volkow et al., 2011; Manchikanti et al., 2012; United States, 2014; United States Centers for Disease Control and Prevention, 2014; King et al., 2014). In recent years, more than four percent of 12-to-21-year-olds qualify as recently active EMPPR users (United States, 2014, 2015). As gauged by expected values based on US experiences between 1980 and 1999, it now is legitimate to speak of a $21^{\text {st }}$ century 'epidemic' of EMPPR use among young people in this country.

60 Underlying fundamental conditions and processes giving rise to this epidemic are being investigated, and new interventions to prevent EMPPR use and to reduce diversion of 
62 legitimately prescribed PPR have been developed (Compton and Volkow, 2006; Cicero and

63 Ellis, 2015; Dart et al., 2015; Kanouse and Compton, 2015; Maxwell, 2015). In addition, once

64 EMPPR use occurs, there is a risk that a syndrome in the form of opioid dependence will occur,

65 as noted elsewhere (Anthony et al., 1994; Martins et al., 2007). In most research projects on this

66 topic, the concept of opioid dependence has been specified in relation to the Fourth Edition of

67 the American Psychiatric Association's Diagnostic and Statistical Manual (American Psychiatric 68 Association, 1994; DSM-IV).

69 One recent contribution to epidemiological evidence about EMPPR use in the United

70 States was made by Meier and colleagues (2012), who discovered a peak risk of becoming a

71 newly incident EMPPR user during the adolescent years from age 12 to 21 . Those estimates were

72 based on data gathered from 2004 to 2008, using what Seedall and Anthony (2014) have called a

73 'mutoscope' approach that can be used to trace the experience of each adolescent birth cohort

74 forward, year by year (Meier et al., 2012). For 12-to-21-year-olds, results for each birth cohort

75 showed peak values of risk at mid-adolescence (roughly age 16 years), followed by declining

76 incidence rates across the later adolescent years (Meier et al., 2012).

77 In this new report on the epidemiology of EMPPR use, also with a focus on 12-to-21-

78 year-olds in the United States, the primary aim is to estimate risk of becoming a newly incident

79 case of DSM-IV-type opioid dependence not too long after EMPPR use starts (i.e., within 12

80 months). We also update EMPPR risk estimates, extending through 2013 the prior 2004-2008

81 estimates of Meier et al. (2012).

82 The value of epidemiological estimates of this type can be seen in their utility as

83 motivators for new or renewed efforts to prevent and control the occurrence of EMPPR use.

84 Estimates of this type also can be used to motivate early outreach and intervention efforts that are 
85 needed to identify and help the new EMPPR users who develop opioid dependence (Compton

86 and Volkow, 2006).

87 


\section{Sidebar 1}

\section{'Extra-Medical' or 'Extramedical' Drug Use in Drug Dependence Epidemiology}

The concepts of 'extra-medical' (or 'extramedical') drug use were introduced in 1989 by a Johns Hopkins University research work group supported by the United States National Institute on Drug Abuse and led by James C. Anthony, then Professor with appointments in the Johns Hopkins University School of Hygiene and Public Health (Mental Hygiene; Epidemiology) and its School of Medicine (Psychiatry and Behavioral Sciences). The research group provided a set of operational specifications for epidemiological field surveys, and proposed a set of pre-written standardized survey items for use in the first United States National Comorbidity Survey (NCS1), scheduled for completion in 1990-92.

By creating a new term, the research group was trying to avoid ambiguities and other problems of signification encountered when terms such as 'drug misuse' and 'non-medical drug use' and 'drug abuse' appeared in the scientific literature. The group thought that these ambiguities and signification problems might be avoided by introducing a completely new term with clear operational specifications.

The team offered this introduction, to be presented to survey participants before its set of proposed standardized survey items on extra-medical drug use:

We are interested in the extra-medical use of these prescription-type drugs.

Extra-medical use is any use on your own; that is, either:

One, without a doctor's prescription, or

Two, in greater amounts than prescribed, or

Three, more often than prescribed, or

Four, for any reasons other than a doctor said you should take them-- such as for kicks, to get high, to feel good, or curiosity about the pill's effect.

The term 'extramedical drug use' first appeared in the peer reviewed scientific literature during 1994, within a journal article on drug dependence epidemiology (Anthony et al. 1994*). This article has been cited more than 1000 times, and at present is cited at a rate of 35-50 citations per year (according to Google Scholar). The term has been adopted by multiple US and international research groups.

*Anthony JC, Warner LA, Kessler RC (1994) Comparative Epidemiology of Dependence on Tobacco, Alcohol, Controlled Substances, and Inhalants: Basic Findings From the National Comorbidity Survey. Experimental and Clinical Psychopharmacology 2(3): 244-268.

A footnote of interest: When the NCS-1 interview schedule was printed, an unnamed member of the NCS-1 field research team decided that 'non-medical' use was intended rather than 'extra-medical' use. As a result, in the NCS-1 codebook, the group's suggested introduction appears with 'non-medical' substituted for 'extra-medical': (http://www.icpsr.umich.edu/cgi-bin/file?comp=none\&study=6693\&ds=1\&file id=946014\&path=NAHDAP, last accessed 8 September 2015. 
89

90

91

92

94

95

96

97

98

100

101

102

103

104

105

106

107

108

109

110

111

\section{MATERIALS \& METHODS}

The study population for this investigation consists of 12-to-21-year-old

noninstitutionalized civilian residents in all 50 states and the District of Columbia during the early $21^{\text {st }}$ century, as sampled and surveyed each year by research teams supported by the US Substance Abuse and Mental Health Services Administration (SAMHSA) during 2002-2013. Each year's community probability sample consisted of survey participants age 12 years and older, after regularized sampling, recruitment, informed consent/assent, and assessment protocols that followed a human subjects protection approach approved by cognizant Institutional Review Boards, as already described in multiple prior reports (e.g., Meier et al., 2012; United States, 2014, 2015; Vsevolozhskaya and Anthony, 2014). The NSDUH sampling frame is noteworthy because it encompasses noninstitutionalized adolescents irrespective of school attendance and wherever they are living, not only in households but also in non-institutional group quarters, dwelling units such as homeless shelters, and college dormitories.

Unweighted numbers of designated 12-21 year old participants in each year's multi-stage area probability sample range from 41,248 to 42,864 (United States, 2013). Survey participation levels for this age group are $\sim 75 \%$ (United States, 2015). Figure S3 provides more details on approximate unweighted sample sizes.

The NSDUH field survey assessment has made use of audio computer assisted selfinterview methods (ACASI), with multiple modules across a range of drug use and health topics, and with coverage of prescription pain relievers via PPR module items listed in Supplement S1. PPR module items have been used to identify newly incident EMPPR users as well as those who had become opioid dependence cases within 12 months after onset of EMPPR use (Martins et al., 2007; Meier et al., 2012; United States, 2015). A generally supportive series of diagnostic 
112 reliability and validity estimates for this type of assessment has been reported by independent

113 research teams (Anthony et al., 1985; Grant et al., 1995; Compton et al., 2013).

114 Whereas NSDUH assessments do not ask about lifetime history of opioid dependence,

115 the survey items have identified newly incident EMPPR users who do and do not qualify as cases

116 of opioid dependence as observed to be present within 12 months after onset of EMPPR use.

117 Based on these assessments, it is possible to identify the newly incident EMPPR users (i.e., those

118 who started EMPPR use within the 12 months prior to assessment), and to measure opioid

119 dependence that occurs after onset of EMPPR use (i.e., within the same 12 month interval).

120 The analysis plan started with Tukey-style exploratory data analysis steps and inspection

121 of analysis-weighted marginal distributions. Thereafter, analysis-weighted estimates of annual

122 incidence rates for EMPPR use were derived, followed by estimation of transition probabilities

123 (with standard errors) for how often opioid dependence cases were observed after onset of

124 EMPPR use (i.e., within 12 months).

125 Our initial attempt to produce year-by-year estimates was thwarted by the small number

126 of opioid dependence cases observed each year, with resulting unstable variance estimates. For

127 this reason, we created year-pairs as well as corresponding age-pairs. This approach produced

128 acceptable stability in the variance estimates. Resulting year-pair estimates for incidence

129 proportions and for the variance of the incidence proportions are appropriate for meta-analysis

130 because each year's sample can serve as a new and statistically independent replication.

131 Accordingly, for meta-analysis purposes, we treated each age-pair and year-pair as an

132 independent replication sample and source of meta-analysis data, building from prior work by

133 Meier et al. (2012) and DeAndrea et al. (2014). 
estimators. When the heterogeneity test statistic suggested potentially important variations (i.e.,

$136 \mathrm{p}<0.05)$, the 'random effects' estimator also is shown. All estimates are analysis-weighted with

137 Taylor series linearization for variance estimation. Meta-analyses are based on Stata Version 13

138 'metan' commands (Stata Corp, 2013), with a logit transformation suggested by Vsevolozhskaya 139 and Anthony (2014).

140 These study estimates might be of special interest to practitioners interested in prevention

141 of opioid dependence, but of course constraints on generalizability deserve mention. Our

142 discussion addresses issues of generalizability, and whether this study's estimates for the nation

143 as a whole might be useful in the context of the work of officials responsible for individual

144 public health districts and states, given what is known about observed state-level variations in the

145 incidence of EMPPR use (Vsevolozhskaya and Anthony, 2014).

\section{RESULTS}

147 Table 1, Panel A, describes the sample of 12-to-21-year-olds. It cross-tabulates effective

148 sample sizes to illustrate unweighted numbers of newly incident EMPPR users in the sample,

149 disclosing peak values between age 14 and age 17 years. Essentially the same peaks are seen in

150 the weighted counts of Panel B of Table 1 and in the analysis-weighted estimates of Table 2.

151 Diagonal cells of these tables also provide what has been called an 'epidemiological

152 mutoscope' view of the experience of individual cohorts. To illustrate, in 2002-3, an estimated

153 1.1-to-1.5 percent of 12-to-13-year-olds had just started EMPPR use. Followed forward to its

154 2004-5 completely independent re-sample, that same cohort had turned 14-15 years old, and

155 cohort-specific risk of EMPPR use had increased to 3.4-to-4.0 percent. Then, with a new re-

156 sample, and observed at age 16-17 years in 2006-7, estimated incidence of EMPPR use for the 
157 same cohort is 4.9-to-5.6 percent, not appreciably distant from the 4.6-to-5.4 percent estimates

158 observed in 2008-9 when the cohort had turned age 18-19 years old. Thereafter, the cohort-

159 specific risk of becoming an EMPPR user dropped to the 2.5-to-3.2 percent level in 2010-11.

160 Followed down its diagonal in Table 2, the cohort-specific pattern for 12-13 year olds in 2004-5

161 is not appreciably different from what can be seen for 12-13 year olds observed in 2002-3.

162 [Seedall and Anthony (2014) provide additional details about this epidemiological mutoscope

163 view of each cohort, which complements what can be learned by studying the row and column

164 totals of each table of this type.]

165 With evidence borrowed from all years, the age-specific meta-analysis summary

166 estimates presented in Table 2 (bottom row) make it clear that no more than about one percent of

167 12-13 year olds became newly incident EMPPR users in these years. The meta-analysis summary

168 estimates disclosed a substantial upward jump in incidence from age 12-13 years to age 14-15

169 years, followed by another substantial jump to peak point estimates at age 16-17 years and age

170 18-19 years, followed by a statistically robust decline in risk for the 20-to-21-year-olds.

Table 2 also might be disclosing a secular trend that merits continuing attention in future

172 years. The peak values for newly incident EMPPR use among 16-to-17-year-olds in 2012-13 are

173 tangibly smaller than corresponding values for prior years, as gauged by non-overlap of

174 confidence intervals (CI). Forest plots presented for age-groups in Figure S5 lead to a similar 175 conclusion.

176 Table 3 shifts attention to risk of becoming opioid dependent within 12 months within

177 onset of EMPPR use. The estimated risk estimates for the age-pair at 14-to-15-years-old is

178 noteworthy in three ways. First, viewed row-wise, except for 2010-11, every year-pair shown in

179 Table 3 (Panel A) discloses a peak point estimate for becoming a case of opioid dependence at 
180 age 14-15 years, Second, meta-analysis summary estimates for the14-to-15-year-old newly

181 incident EMPPR users are robustly larger than estimates observed for the other age-pairs. Third,

182 with no more than one exception, the epidemiological mutoscope view of the table's diagonal

183 cells shows that peak incidence of opioid dependence for birth cohorts (given newly incident

184 EMPPR use) is larger at age 14-15 years than at other ages.

185 The epidemiological estimates presented in Tables 2 and 3 seem to be quite clear in their

186 implications for age-specific timing of public health interventions. Delay of outreach programs

187 and interventions until the adult years, with failure to concentrate resources on teenagers, might

188 well be misguided, if these incidence estimates are judged to be trustworthy and actionable.

189 For a variety of reasons, including the technical detail known as right-censoring in the

190 context of survival analyses, it can be difficult to estimate the mean duration of EMPPR use. By

191 combining this study's annual incidence estimates with separately published prevalence

192 estimates (United States, 2006-2015), it is possible to derive such estimates for EMPPR use via a

193 functional relationship that expresses prevalence as a function of incidence times mean duration.

194 Formulated in this fashion, when EMPPR use starts during the adolescent years, its mean

195 duration can be estimated as 2-4 years. Tables S6 and S7 provide details about these calculations.

\section{DISCUSSION \& CONCLUSIONS}

197 For scientists who study prescription pain relievers, policy-makers, regulatory bodies and

198 health professionals, this report's characterization of a $21^{\text {st }}$ century 'epidemic' may be useful.

199 Consistent with prior estimates through 2008 (e.g., Meier et al., 2012), years of peak risk for

200 starting extra-medical PPR use are observed during mid-adolescence.

201 When this study's epidemiological estimates are applied to population counts for 12-21

202 year olds in the US, the calculations suggest that roughly 8 million adolescents started using PPR 
203 extra-medically between 2002 and 2013. In addition, during each year between 2002 and 2013,

204 roughly 42,000 to 58,000 became newly incident cases of opioid dependence within 12 months

205 after onset of extra-medical use (i.e., at least 120 cases per day). One can presume that all or

206 most of these cases might be in need of advice or monitoring by a general practitioner, if not

207 more intensive drug treatment services.

At $2.4 \%(95 \% \mathrm{CI}=1.7 \%, 3.4 \%)$, the estimated risk for becoming an opioid dependence case within 12 months after onset of EMPPR use is relatively low among 20-to-21-year-olds.

210 Opioid dependence risk estimates seen for newly incident 12-to-17-year-old EMPPR users are 2-

2113 times larger, possibly reflecting a more general susceptibility to complications when drug use

212 starts early, as seen elsewhere (e.g., Anthony \& Petronis, 1995). An apparent peak risk for

213 transition to opioid dependence within 12 months at age 14-15 years is noteworthy. In the US, by

214 age 14-15 years, most teenagers have qualified for admission to the secondary school level.

215 Perhaps PPR become more readily available once primary school years have passed.

216 In this report, by joining previously published age-specific estimates for prevalence of

217 EMPPR use with this study's newly published age-specific estimates of incidence rates through

218 2013, it has been possible to discover that for the most part the mean duration of extra-medical

219 PPR use is on the order of 2-4 years, although an allowance for age-related variation must be

220 made. Previously published estimates suggest that many EMPPR users try these compounds no

221 more than a few times and then stop, with duration far shorter than the estimated mean, whereas

222 others become persistent users, with duration considerably longer than the estimated mean (e.g.,

223 those who become opioid dependence cases). We regret that statistically reliable age-specific

224 estimates for the duration of opioid dependence attributable to PPR cannot be derived from these

225 NSDUH data. 
227 PPR use and dependence, can use this study's estimates in attempts to marshal new resources for

228 clinical and population health initiatives. Estimates of this type might help motivate design,

229 implementation, and evaluation of more effective public health outreach and early intervention

230 services for adolescent-onset users and opioid dependence cases in the community.

231 Among study limitations, we note the self-report character of NSDUH data, for which we

232 have no logistically feasible alternative in nationally representative community sample surveys.

233 While it is true that ACASI assessment protocols qualify as 'best practices' for large sample

234 quantitative survey research on generally illegal and sensitive behaviors, some young people in

235 these samples might fail to disclose newly incident EMPPR use or clinical features of opioid

236 dependence once it occurs (i.e., lapses in field assessment 'sensitivity'). In addition, not all

237 EMPPR users are assessed exactly one year after onset of EMPPR use. As such, this study's

238 estimates fall somewhat short of 'annual' incidence rates. R-DAS estimation does not make it

239 possible to calculate person-months from first day of EMPPR use to NSDUH assessment dates

240 (United States, 2014). In addition, the technical detail of post-stratification adjustment to US

241 Census cell counts is allowed to vary across R-DAS year pairs. In consequence, comparisons

242 across NSDUH year-pairs might show variations due to variations in the US Census cell counts.

243 As such, some degree of caution is needed when comparing R-DAS estimates across year-pairs.

244 With respect to the measurement issues just noted, a lack of specificity might counter-

245 balance departures from 100\% sensitivity. Some adolescents might exaggerate and boast about

246 EMPPR experiences that never truly occurred. Alternately, some might misunderstand survey

247 questions in a direction that creates specificity errors. Therefore, as is true for almost all

248 epidemiological surveillance estimates, the large-sample scale for survey coverage required to 
249 achieve nationally representative probability samples tends to thwart deep probes into qualitative

250 research issues of screening or diagnostic validity as might be achieved via drug toxicology

251 assays or a standardized clinical reappraisal work-up of a type made feasible in research with

252 smaller and more local samples. Nonetheless, in more than 30 years of standardized clinical

253 reappraisal research on large sample survey-based diagnostic assessments of DSM-type drug

254 dependence, validity evidence has generally been supportive, often more supportive than has

255 been true for other DSM categories of neuropsychiatric disorders, as noted in articles cited

256 within our Methods section.

257 In any community sample survey of drug outcomes, there is a possibility of omissions of

258 seriously disabled opioid-dependent users with lives so disrupted that they qualify as non-

259 participants in the survey operations, even when their names are included on community survey

260 sampling rosters. This is a methodological issue that pertains to the sensitivity of the surveillance

261 operation, and is not an issue of the sensitivity of the survey measurements per se. To the extent

262 that this research project involves estimation of the probability of transitioning from first onset of

263 use into opioid dependence, there might a slight under-estimation of these probabilities, due to

264 left-censoring of severely affected cases of this type. A topic of continuing investigation, not yet

265 resolved, is how often users move quickly from first use into dependence syndromes that are

266 disabling to the point of survey non-participation. Our expectation is for a small downward bias

267 in the estimates, given that they generally are based upon users in the earliest months of the

268 opioid dependence process. A related omission involves fatal overdose deaths as might occur on

269 the first or second use of the drug, in which case these users can be missing from the survey

270 denominators altogether. US vital statistics, to date, suggest that no more than a handful of such

271 deaths would be missed by NSDUH field survey operations, given that the number of 
272 prescription opioid overdose deaths in the US totals no more than about 16,000 for the country as

273 a whole, with a total population size of roughly 320 million individuals (United States Centers

274 for Disease Control and Prevention, 2014). A recent study of the potential 'Len Bias' bias

275 suggests negligible error from this theoretically interesting source of epidemiological bias

276 (Lopez-Quintero et al., 2015).

277 Readers interested in dependence that occurs after strictly medical use of PPR may be 278 disappointed that NSDUH focuses exclusively on extra-medical PPR use. One must look 279 elsewhere for opioid dependence estimates when users stay well within boundaries of a 280 prescribing clinician's instructions. It seems reasonable that this study's estimates of opioid 281 dependence transition probabilities might be larger than what would be observed in research on

282 PPR use exactly as prescribed, because the susceptibility traits giving rise to adolescent-onset 283 extra-medical drug use almost certainly overlap with those influencing adolescent-onset drug 284 dependence. Nonetheless, there now are no definitive nation-level estimates for these transition 285 probabilities in the context of medically prescribed use. Estimates of this type will be needed to 286 make a direct comparison of dependence risks across these different contexts of medical and

287 EMPPR use. We acknowledge that the relationship between PPR use and opioid dependence can 288 be complex, with feedback loops. Quite clearly, EM use can lead to dependence, but dependence 289 that develops during medically prescribed use also can lead to EMPPR use, as discussed 290 elsewhere (e.g., Anthony, 2010).

Another question is whether these estimates for the US as a whole might generalize to 292 sub-units as small as public health districts, to jurisdictions as large as states or regions within 293 the US, or to other countries. There is substantial evidence of state-level variations in the 294 occurrence of EMPPR use (Vsevolozhskaya and Anthony, 2014). Observed variations in those 
295 estimates lead us to hesitate before recommending application of this study's estimates to state or 296 sub-state units. Fortunately, R-DAS 10-year datasets (2002-2011) make provisions for sub-state

297 estimates. For the larger sub-states (and for states), it is possible to pool data across 10 years in

298 order to derive summary estimates. As for applicability elsewhere (i.e., in other countries), we 299 urge caution.

300 Notwithstanding limitations of this type, and the fact that the transition probabilities for 301 onset of opioid dependence within 12 months actually might be somewhat larger than we have 302 estimated, the epidemiological estimates observed here are not trivial. These estimates deserve 303 both clinical and public health attention. These estimates should help encourage and motivate the 304 US National Institute on Drug Abuse, Centers for Disease Prevention and Control, and others to 305 focus or renew attention to 12-21 year olds in current efforts to prevent and control extra-medical 306 use of prescription pain relievers and associated opioid dependence risks. 
308

309

310

311

312

313

314

315

316

317

318

319

320

321

322

323

324

325

326

327

328

329

330

331

\section{REFERENCES}

American Psychiatric Association (1994) DSM-IV: Diagnostic and Statistical Manual of Mental Disorders (4th edition). Washington, DC: American Psychiatric Association

Anthony JC (2010) Novel phenotype issues raised in cross-national epidemiological research on drug dependence. Ann N Y Acad Sci 1187: 353-369. doi: 10.1111/j.17496632.2009.05419.x

Anthony JC, Folstein M, Romanoski AJ, Von Korff MR, Nestadt GR, Chahal R, Merchant A, Brown CH, Shapiro S, Kramer M, et al. (1985) Comparison of the lay Diagnostic Interview Schedule and a standardized psychiatric diagnosis. Experience in eastern Baltimore. Arch Gen Psychiatry 42(7), 667-675

Anthony J C, Petronis KR (1995) Early-onset drug use and risk of later drug problems. Drug Alcohol Depend 40(1), 9-15

Anthony JC, Warner LA, Kessler RC (1994) Comparative epidemiology of dependence on tobacco, alcohol, controlled substances, and inhalants: Basic findings from the National Comorbidity Survey. Exp Clin Psychopharmacol 2(3), 244-268. http://doi.org/10.1037/1064-1297.2.3.244

Cicero TJ, Ellis MS (2015) Abuse-Deterrent Formulations and the Prescription Opioid Abuse Epidemic in the United States: Lessons Learned From OxyContin. JAMA Psychiatry, 72(5), 424-430. http://doi.org/10.1001/jamapsychiatry.2014.3043

Compton WM, Dawson DA, Goldstein RB, Grant BF (2013) Crosswalk between DSM-IV dependence and DSM-5 substance use disorders for opioids, cannabis, cocaine and alcohol. Drug Alcohol Depend 132(1-2), 387-390. http://doi.org/10.1016/j.drugalcdep.2013.02.036 
332 Compton WM, Volkow ND (2006) Major increases in opioid analgesic abuse in the United

333 States: concerns and strategies. Drug Alcohol Depend 81(2), 103-107.

334 http://doi.org/10.1016/j.drugalcdep.2005.05.009

335 Dart RC, Surratt HL, Cicero TJ, Parrino MW, Severtson SG, Bucher-Bartelson B, Green, JL 336 (2015) Trends in Opioid Analgesic Abuse and Mortality in the United States. N Engl J Med, 372(3), 241-248. http://doi.org/10.1056/NEJMsa1406143

DeAndrea DC, Troost JP, Anthony JC (2013) Toward primary prevention of extra-medical OxyContin ${ }^{\circledR}$ use among young people. Prev Med 57(3), 244-246 http://doi.org/10.1016/j.ypmed.2013.05.004

341 Grant BF, Harford TC, Dawson DA, Chou PS, Pickering RP (1995) The alcohol use disorder and associated disabilities interview schedule (AUDADIS): reliability of alcohol and drug modules in a general population sample. Drug Alcohol Depend 39(1), 37-44. http://doi.org/10.1016/0376-8716(95)01134-K. Mortality among heroin users and users of other internationally regulated drugs: A 27year follow-up of users in the Epidemiologic Catchment Area Program household samples. Drug and Alcohol Depend. 2015 September 3 doi: 10.1016/j.drugalcdep.2015.08.030. [Epub ahead of print].

Kanouse AB, Compton P (2015) The Epidemic of Prescription Opioid Abuse, the Subsequent Rising Prevalence of Heroin Use, and the Federal Response. J Pain Palliat Care Pharmacother, 29(2), 102-114. http://doi.org/10.3109/15360288.2015.1037521

King NB, Fraser V, Boikos C, Richardson R, Harper S (2014) Determinants of increased opioidrelated mortality in the United States and Canada, 1990-2013: a systematic review. Am J 
Public Health 104(8), e32-42. http://doi.org/10.2105/AJPH.2014.301966

356

357

358

359

360

361

362

363

364

365

366

367

368

369

370

371

372

373

374

375

Manchikanti L, Helm S, Fellows B, Janata JW, Pampati V, Grider JS, Boswell MV (2012)

Opioid epidemic in the United States. Pain Physician 15(3 Suppl), ES9-38

Martins SS, Ghandour LA, Chilcoat HD (2007) Profile of dependence symptoms among extramedical opioid analgesic users. Addict Behav 32(10), 2003-2019. http://doi.org/10.1016/j.addbeh.2007.01.006

Maxwell JC (2015) The Pain Reliever and Heroin Epidemic in the United States: Shifting Winds in the Perfect Storm. J Addict Dis. 2015 Jun 24:0 [Epub ahead of print]. http://doi.org/10.1080/10550887.2015.1059667

Meier EA, Troost JP, Anthony JC (2012) Extramedical use of prescription pain relievers by youth aged 12 to 21 years in the United States: national estimates by age and by year. Arch Pediatr Adolesc Med 166(9), 803-807. http://doi.org/10.1001/archpediatrics.2012.209

Parker MA, Anthony JC (2014) Should anyone be riding to glory on the now-descending limb of the crack-cocaine epidemic curve in the United States? Drug Alcohol Depend 138, 225228. http://doi: 10.1016/j.drugalcdep.2014.02.005

Seedall RB, Anthony JC (2014) Monitoring by Parents and Hypothesized Male-Female Differences in Evidence from a Nationally Representative Cohort Re-sampled from Age 12 to 17 Years: An Exploratory Study Using a "Mutoscope" Approach. Prev Sci 1-11. http://doi.org/10.1007/s11121-014-0517-8

StataCorp (2013) Stata Statistical Software: Release 13. College Station, TX: StataCorp LP 
376 United States (2006) The NSDUH Report - Nonmedical Users of Pain Relievers: Characteristics

377 of Recent Initiates. Substance Abuse and Mental Health Services Administration, Office

$378 \quad$ of Applied Studies

379 United States (2012) Results from the 2011 National Survey on Drug Use and Health: Summary

380 of National Findings. NSDUH Series H-44, HHS Publication No. (SMA) 12-4713.

381 Rockville, MD: Substance Abuse and Mental Health Services Administration

382 United States (2013) Results from the 2013 National Survey on Drug Use and Health: Detailed

383 Tables. Substance Abuse and Mental Health Services Administration.

384 United States (2014) Results from the 2013 National Survey on Drug Use and Health: Summary

385 of National Findings. NSDUH Series H-48, HHS Publication No. (SMA) 14-4863.

386 Rockville: Substance Abuse and Mental Health Services Administration

387 United States (2015) National Survey on Drug Use and Health: 2-Year R-DAS (2002 to 2003,

3882004 to 2005,2006 to 2007,2008 to 2009, 2010 to 2011, and 2012 to 2013). Department

389 of Health and Human Services. ICPSR34482-v3. Ann Arbor, MI: Inter-university

390 Consortium for Political and Social Research [distributor].

$391 \quad$ http://doi.org/10.3886/ICPSR34482.v3

392 United States Centers for Disease Control and Prevention (2014) Prescription Drug Overdose in

393 the United States: Fact Sheet. Available via

394 http://www.cdc.gov/homeandrecreationalsafety/overdose/facts.html. Accessed 15 Sept

$395 \underline{2014}$

396 Volkow ND, McLellan TA, Cotto JH, Karithanom M, Weiss SRB (2011) Characteristics of

397 opioid prescriptions in 2009. JAMA 305(13), 1299-1301.

398 http://doi.org/10.1001/jama.2011.401 
399 Vsevolozhskaya OA, Anthony JC (2014) Confidence interval estimation in R-DAS. Drug Alcohol Depend 143(10) 95-104. http://doi.org/10.1016/j.drugalcdep.2014.07.017.

401 
402 Table 1. Approximate unweighted numbers of newly incident adolescent onset extra-medical 403 users of prescription pain relievers per subgroup (Panel A) and weighted population counts 404 (Panel B) for newly incident extra-medical prescription pain reliever users by age and year-pair. 405 Data from Restricted-use Data Analysis System subsamples of the National Surveys on Drug 406 Use and Heath, United States 2002-2013.

407 408

Table 1, Panel A: Approximate number of newly incident users in the sample

\begin{tabular}{llllll}
\hline Year pair & $\mathbf{1 2 - 1 3} \mathbf{y}$ & $\mathbf{1 4 - 1 5} \mathbf{y}$ & $\mathbf{1 6 - 1 7} \mathbf{y}$ & $\mathbf{1 8 - 1 9} \mathbf{y}$ & $\mathbf{2 0 - 2 1} \mathbf{y}$ \\
\hline $2002-2003$ & 191 & 625 & 814 & 564 & 308 \\
\hline $2004-2005$ & 199 & 520 & 732 & 477 & 303 \\
\hline $2006-2007$ & 171 & 476 & 681 & 532 & 263 \\
\hline $2008-2009$ & 145 & 546 & 708 & 514 & 285 \\
\hline $2010-2011$ & 157 & 446 & 679 & 426 & 249 \\
\hline $2012-2013$ & 118 & 353 & 507 & 313 & 260 \\
\hline
\end{tabular}

Table 1, Panel B: Corresponding weighted count of newly incident users in the US population

\begin{tabular}{llllll}
\hline Year pair & $\mathbf{1 2 - 1 3} \mathbf{y}$ & $\mathbf{1 4 - 1 5} \mathbf{y}$ & $\mathbf{1 6 - 1 7} \mathbf{y}$ & $\mathbf{1 8 - 1 9} \mathbf{y}$ & $\mathbf{2 0 - 2 1} \mathbf{y}$ \\
\hline $2002-2003$ & 104,000 & 344,000 & 454,000 & 397,000 & 222,000 \\
\hline $2004-2005$ & 113,000 & 301,000 & 430,000 & 351,000 & 224,000 \\
\hline $2006-2007$ & 98,000 & 276,000 & 397,000 & 418,000 & 205,000 \\
\hline $2008-2009$ & 81,000 & 313,000 & 408,000 & 392,000 & 215,000 \\
\hline $2010-2011$ & 86,000 & 248,000 & 378,000 & 329,000 & 196,000 \\
\hline $2012-2013$ & 66,000 & 202,000 & 290,000 & 254,000 & 208,000 \\
\hline
\end{tabular}

$\begin{array}{llllll}\text { Estimated } & 548,000 & 1,684,000 & 2,357,000 & 2,141,000 & 1,270,000\end{array}$

analysis-weighted total (per 100) 
410 Table 2. Estimated risk of becoming a newly incident extra-medical user of prescription pain 411 relievers, stratified by age at assessment and survey year-pair. Age- and time-specific incidence 412 estimates (Panel A), 95\% confidence intervals (Panel B), and age-specific meta-analysis

413 summary estimates. Data from Restricted-use Data Analysis System subsamples of the National

414 Surveys on Drug Use and Heath, United States 2002-2013.

415

Table 2, Panel A: Estimated risk of becoming a newly incident user (per 100)

\begin{tabular}{llllll} 
Year pair & $\mathbf{1 2 - 1 3} \mathbf{y}$ & $\mathbf{1 4 - 1 5} \mathbf{y}$ & $\mathbf{1 6 - 1 7} \mathbf{y}$ & $\mathbf{1 8 - 1 9} \mathbf{y}$ & $\mathbf{2 0 - 2 1} \mathbf{y}$ \\
\hline $2002-2003$ & 1.3 & 4.4 & 6.3 & 5.7 & 3.5 \\
\hline $2004-2005$ & 1.4 & 3.7 & 5.9 & 4.9 & 3.7 \\
\hline $2006-2007$ & 1.2 & 3.4 & 5.2 & 5.6 & 3.3 \\
\hline $2008-2009$ & 1.1 & 3.9 & 5.3 & 5.0 & 3.4 \\
\hline $2010-2011$ & 1.1 & 3.2 & 4.9 & 4.3 & 2.8 \\
\hline $2012-2013$ & 0.8 & 2.5 & 3.8 & 3.3 & 3.0 \\
\hline
\end{tabular}

Table 2, Panel B: 95\% Confidence Intervals for Estimates in Panel A (per 100)

\begin{tabular}{llllll}
\hline Year pair & $\mathbf{1 2 - 1 3} \mathbf{y}$ & $\mathbf{1 4 - 1 5} \mathbf{~}$ & $\mathbf{1 6 - 1 7} \mathbf{y}$ & $\mathbf{1 8 - 1 9} \mathbf{y}$ & $\mathbf{2 0 - 2 1} \mathbf{~}$ \\
\hline $2002-2003$ & $1.1,1.5$ & $4.1,4.8$ & $5.9,6.8$ & $5.2,6.2$ & $3.1,3.9$ \\
\hline $2004-2005$ & $1.2,1.6$ & $3.4,4.0$ & $5.5,6.3$ & $4.5,5.4$ & $3.3,4.1$ \\
\hline $2006-2007$ & $1.1,1.4$ & $3.1,3.7$ & $4.9,5.6$ & $5.2,6.1$ & $2.9,3.7$ \\
\hline $2008-2009$ & $0.9,1.3$ & $3.6,4.2$ & $4.9,5.7$ & $4.6,5.4$ & $3.1,3.9$ \\
\hline $2010-2011$ & $1.0,1.3$ & $2.9,3.5$ & $4.6,5.3$ & $3.9,4.7$ & $2.5,3.2$ \\
\hline $2012-2013$ & $0.7,1.1$ & $2.2,2.9$ & $3.4,4.2$ & $2.9,3.8$ & $2.5,3.5$ \\
\hline
\end{tabular}

Meta-analysis summary estimates \& $95 \%$ confidence intervals (per 100) $1.2(1.0,1.3) \quad 3.5(3.0,4.0) \quad 5.2(4.5,5.9) \quad 4.8(4.1,5.5) \quad 3.3(3.1,3.5)^{\mathrm{b}}$

417

418

419

420

421

422

423

a Supplement S4 provides additional information about I-squared.

b Here, the I-squared statistic has $0.05>p>0.15$ so the $95 \%$ CI are from 'fixed effects' estimation; the corresponding 'random effects' interval is 3.0, 3.6. All other meta-analytic 95\% $\mathrm{CI}$ are from random effects estimation (due to I-squared $\mathrm{p}<0.05$ ). 
424 Table 3. Estimated risk of transitioning and becoming an opioid dependence case no longer than 42512 months after onset of starting to use prescription pain relievers extra-medically (Panel A), 426 95\% confidence intervals (Panel B), and age-specific meta-analysis summary estimates. Data 427 from Restricted-use Data Analysis System subsamples of the National Surveys on Drug Use and 428 429

Heath, United States 2002-2013.

Table 3, Panel A: Estimated risk of becoming an opioid dependence case (per 100 newly incident EMPPR users)

\begin{tabular}{llllll}
\hline Year pair & $\mathbf{1 2 - 1 3} \mathbf{y}$ & $\mathbf{1 4 - 1 5} \mathbf{y}$ & $\mathbf{1 6 - 1 7} \mathbf{y}$ & $\mathbf{1 8 - 1 9} \mathbf{y}$ & $\mathbf{2 0 - 2 1} \mathbf{y}$ \\
\hline $2002-2003$ & 4.2 & 6.3 & 4.7 & 2.8 & 1.8 \\
\hline $2004-2005$ & 3.2 & 9.8 & 5.1 & 3.3 & 2.4 \\
\hline $2006-2007$ & 5.2 & 7.9 & 3.9 & 1.7 & 3.4 \\
\hline $2008-2009$ & 5.4 & 7.5 & 4.8 & 4.2 & 3.2 \\
\hline $2010-2011$ & 8.2 & 5.8 & 7.1 & 6.1 & 2.4 \\
\hline $2012-2013$ & 5.1 & 6.4 & 6.1 & 4.8 & 2.1 \\
\hline
\end{tabular}

Table 3, Panel B: 95\% Confidence Intervals for Panel A estimates (per 100)

\begin{tabular}{llllll}
\hline Year pair & $\mathbf{1 2 - 1 3} \mathbf{y}$ & $\mathbf{1 4 - 1 5} \mathbf{y}$ & $\mathbf{1 6 - 1 7} \mathbf{y}$ & $\mathbf{1 8 - 1 9} \mathbf{y}$ & $\mathbf{2 0 - 2 1} \mathbf{~}$ \\
\hline $2002-2003$ & $2.3,7.4$ & $4.3,9.1$ & $3.1,7.1$ & $1.5,5.1$ & $0.9,3.7$ \\
\hline $2004-2005$ & $1.7,6.0$ & $6.9,13.6$ & $3.4,7.6$ & $1.8,5.9$ & $1.1,5.0$ \\
\hline $2006-2007$ & $2.8,9.7$ & $5.2,11.7$ & $2.3,6.5$ & $0.8,3.4$ & $1.5,7.7$ \\
\hline $2008-2009$ & $2.6,11.0$ & $5.4,10.4$ & $3.4,6.7$ & $2.4,7.3$ & $1.3,7.8$ \\
\hline $2010-2011$ & $4.4,14.7$ & $3.6,9.2$ & $4.7,10.7$ & $3.4,10.7$ & $0.9,6.1$ \\
\hline $2012-2013$ & $2.6,15.1$ & $3.8,10.4$ & $3.9,9.5$ & $2.4,9.2$ & $0.8,5.2$ \\
\hline
\end{tabular}

Meta-analysis

summary estimates

\& $95 \%$ confidence

$5.1(3.9,6.6)$

$7.4(6.3,8.7)$

$5.2(4.4,6.2)$

$3.6(2.5,5.0)^{\mathrm{b}} \quad 2.4(1.7,3.4)$

intervals (per 100)

432

433

434

435

436

437 a Supplement S4 provides additional information about I-squared.

${ }^{b}$ Here, the I-squared statistic has $0.05<p<0.15$ so the $95 \%$ CI are from 'random effects' estimation; the corresponding 'fixed effects' interval is 2.8, 4.6. All other meta-analytic 95\% CI are from 'fixed effects' estimation (due to I-squared $\mathrm{p}>0.15$ ). 


\section{Table $\mathbf{1}$ (on next page)}

Approximate unweighted numbers of newly incident adolescent onset extra-medical users of prescription pain relievers per subgroup and weighted population counts by age and year-pair.

Approximate unweighted numbers (Panel A) and weighted population counts (Panel B) for newly incident extra-medical prescription pain reliever users. Data from Restricted-use Data Analysis System subsamples of the National Surveys on Drug Use and Heath, United States 2002-2013. 
1 Table 1, Panel A: Approximate number of newly incident users in the sample

\begin{tabular}{llllll}
\hline Year pair & $\mathbf{1 2 - 1 3} \mathbf{y}$ & $\mathbf{1 4 - 1 5} \mathbf{y}$ & $\mathbf{1 6 - 1 7} \mathbf{y}$ & $\mathbf{1 8 - 1 9} \mathbf{y}$ & $\mathbf{2 0 - 2 1} \mathbf{y}$ \\
\hline $2002-2003$ & 191 & 625 & 814 & 564 & 308 \\
\hline $2004-2005$ & 199 & 520 & 732 & 477 & 303 \\
\hline $2006-2007$ & 171 & 476 & 681 & 532 & 263 \\
\hline $2008-2009$ & 145 & 546 & 708 & 514 & 285 \\
\hline $2010-2011$ & 157 & 446 & 679 & 426 & 249 \\
\hline $2012-2013$ & 118 & 353 & 507 & 313 & 260 \\
\hline
\end{tabular}

Table 1, Panel B: Corresponding weighted count of newly incident users in the US population

\begin{tabular}{llllll} 
Year pair & $\mathbf{1 2 - 1 3} \mathbf{y}$ & $\mathbf{1 4 - 1 5} \mathbf{y}$ & $\mathbf{1 6 - 1 7} \mathbf{y}$ & $\mathbf{1 8 - 1 9} \mathbf{y}$ & $\mathbf{2 0 - 2 1} \mathbf{y}$ \\
\hline $2002-2003$ & 104,000 & 344,000 & 454,000 & 397,000 & 222,000 \\
\hline $2004-2005$ & 113,000 & 301,000 & 430,000 & 351,000 & 224,000 \\
\hline $2006-2007$ & 98,000 & 276,000 & 397,000 & 418,000 & 205,000 \\
\hline $2008-2009$ & 81,000 & 313,000 & 408,000 & 392,000 & 215,000 \\
\hline $2010-2011$ & 86,000 & 248,000 & 378,000 & 329,000 & 196,000 \\
\hline $2012-2013$ & 66,000 & 202,000 & 290,000 & 254,000 & 208,000 \\
\hline
\end{tabular}

$\begin{array}{llllll}\text { Estimated } & 548,000 & 1,684,000 & 2,357,000 & 2,141,000 & 1,270,000\end{array}$




\section{Table 2 (on next page)}

Estimated risk of becoming a newly incident extra-medical user of prescription pain relievers, stratified by age at assessment and survey year-pair.

Age- and time-specific incidence estimates (Panel A), 95\% confidence intervals (Panel B), and age-specific meta-analysis summary estimates. Data from Restricted-use Data Analysis System subsamples of the National Surveys on Drug Use and Heath, United States 20022013. 
1 Table 2, Panel A: Estimated risk of becoming a newly incident user (per 100)

\begin{tabular}{llllll}
\hline Year pair & $\mathbf{1 2 - 1 3} \mathbf{y}$ & $\mathbf{1 4 - 1 5} \mathbf{y}$ & $\mathbf{1 6 - 1 7} \mathbf{y}$ & $\mathbf{1 8 - 1 9} \mathbf{y}$ & $\mathbf{2 0 - 2 1} \mathbf{y}$ \\
\hline $2002-2003$ & 1.3 & 4.4 & 6.3 & 5.7 & 3.5 \\
\hline $2004-2005$ & 1.4 & 3.7 & 5.9 & 4.9 & 3.7 \\
\hline $2006-2007$ & 1.2 & 3.4 & 5.2 & 5.6 & 3.3 \\
\hline $2008-2009$ & 1.1 & 3.9 & 5.3 & 5.0 & 3.4 \\
\hline $2010-2011$ & 1.1 & 3.2 & 4.9 & 4.3 & 2.8 \\
\hline $2012-2013$ & 0.8 & 2.5 & 3.8 & 3.3 & 3.0
\end{tabular}

Table 2, Panel B: 95\% Confidence Intervals for Estimates in Panel A (per 100)

\begin{tabular}{llllll}
\hline Year pair & $\mathbf{1 2 - 1 3} \mathbf{y}$ & $\mathbf{1 4 - 1 5} \mathbf{y}$ & $\mathbf{1 6 - 1 7} \mathbf{y}$ & $\mathbf{1 8 - 1 9} \mathbf{y}$ & $\mathbf{2 0 - 2 1} \mathbf{y}$ \\
\hline $2002-2003$ & $1.1,1.5$ & $4.1,4.8$ & $5.9,6.8$ & $5.2,6.2$ & $3.1,3.9$ \\
\hline $2004-2005$ & $1.2,1.6$ & $3.4,4.0$ & $5.5,6.3$ & $4.5,5.4$ & $3.3,4.1$ \\
\hline $2006-2007$ & $1.1,1.4$ & $3.1,3.7$ & $4.9,5.6$ & $5.2,6.1$ & $2.9,3.7$ \\
\hline $2008-2009$ & $0.9,1.3$ & $3.6,4.2$ & $4.9,5.7$ & $4.6,5.4$ & $3.1,3.9$ \\
\hline $2010-2011$ & $1.0,1.3$ & $2.9,3.5$ & $4.6,5.3$ & $3.9,4.7$ & $2.5,3.2$ \\
\hline $2012-2013$ & $0.7,1.1$ & $2.2,2.9$ & $3.4,4.2$ & $2.9,3.8$ & $2.5,3.5$ \\
\hline
\end{tabular}

Meta-analysis

summary estimates

\& 95\% confidence $\quad 1.2(1.0,1.3) \quad 3.5(3.0,4.0) \quad 5.2(4.5,5.9) \quad 4.8(4.1,5.5) \quad 3.3(3.1,3.5)^{b}$ intervals (per 100)

3 a Supplement S4 provides additional information about I-squared.

$4{ }^{b}$ Here, the I-squared statistic has $0.05>p>0.15$ so the $95 \%$ CI are from 'fixed effects'

5 estimation; the corresponding 'random effects' interval is 3.0, 3.6. All other meta-analytic 95\%

$6 \mathrm{CI}$ are from random effects estimation (due to I-squared $\mathrm{p}<0.05$ ). 


\section{Table 3 (on next page)}

Estimated risk of transitioning and becoming an opioid dependence case no longer than 12 months after onset of starting to use prescription pain relievers extra-medically.

Estimated risk of transitioning to opioid dependence (Panel A), 95\% confidence intervals (Panel B), and age-specific meta-analysis summary estimates. Data from Restricted-use Data Analysis System subsamples of the National Surveys on Drug Use and Heath, United States 2002-2013. 
1 Table 3, Panel A: Estimated risk of becoming an opioid dependence case (per 100 newly

2 incident EMPPR users)

\begin{tabular}{llllll}
\hline Year pair & $\mathbf{1 2 - 1 3} \mathbf{y}$ & $\mathbf{1 4 - 1 5} \mathbf{y}$ & $\mathbf{1 6 - 1 7} \mathbf{y}$ & $\mathbf{1 8 - 1 9} \mathbf{y}$ & $\mathbf{2 0 - 2 1} \mathbf{y}$ \\
\hline $2002-2003$ & 4.2 & 6.3 & 4.7 & 2.8 & 1.8 \\
\hline $2004-2005$ & 3.2 & 9.8 & 5.1 & 3.3 & 2.4 \\
\hline $2006-2007$ & 5.2 & 7.9 & 3.9 & 1.7 & 3.4 \\
\hline $2008-2009$ & 5.4 & 7.5 & 4.8 & 4.2 & 3.2 \\
\hline $2010-2011$ & 8.2 & 5.8 & 7.1 & 6.1 & 2.4 \\
\hline $2012-2013$ & 5.1 & 6.4 & 6.1 & 4.8 & 2.1 \\
\hline
\end{tabular}

Table 3, Panel B: 95\% Confidence Intervals for Panel A estimates (per 100)

\begin{tabular}{llllll}
\hline Year pair & $\mathbf{1 2 - 1 3} \mathbf{y}$ & $\mathbf{1 4 - 1 5} \mathbf{y}$ & $\mathbf{1 6 - 1 7} \mathbf{y}$ & $\mathbf{1 8 - 1 9} \mathbf{y}$ & $\mathbf{2 0 - 2 1} \mathbf{y}$ \\
\hline $2002-2003$ & $2.3,7.4$ & $4.3,9.1$ & $3.1,7.1$ & $1.5,5.1$ & $0.9,3.7$ \\
\hline $2004-2005$ & $1.7,6.0$ & $6.9,13.6$ & $3.4,7.6$ & $1.8,5.9$ & $1.1,5.0$ \\
\hline $2006-2007$ & $2.8,9.7$ & $5.2,11.7$ & $2.3,6.5$ & $0.8,3.4$ & $1.5,7.7$ \\
\hline $2008-2009$ & $2.6,11.0$ & $5.4,10.4$ & $3.4,6.7$ & $2.4,7.3$ & $1.3,7.8$ \\
\hline $2010-2011$ & $4.4,14.7$ & $3.6,9.2$ & $4.7,10.7$ & $3.4,10.7$ & $0.9,6.1$ \\
\hline $2012-2013$ & $2.6,15.1$ & $3.8,10.4$ & $3.9,9.5$ & $2.4,9.2$ & $0.8,5.2$
\end{tabular}

Meta-analysis

summary estimates

$\& 95 \%$ confidence

$5.1(3.9,6.6) \quad 7.4(6.3,8.7) \quad 5.2(4.4,6.2) \quad 3.6(2.5,5.0)^{\mathrm{b}} \quad 2.4(1.7,3.4)$

intervals $(\text { per 100) })^{\mathrm{a}}$

3

$4 \quad{ }^{a}$ Supplement S4 provides additional information about I-squared.

5 b Here, the I-squared statistic has $0.05<\mathrm{p}<0.15$ so the $95 \% \mathrm{CI}$ are from 'random effects'

6 estimation; the corresponding 'fixed effects' interval is 2.8, 4.6. All other meta-analytic 95\% CI

7 are from 'fixed effects' estimation (due to I-squared $\mathrm{p}>0.15$ ). 